\title{
Reproducibility improves exponentially over 63 years of social learning research
}

\author{
Riana Minocher ${ }^{1}$, Silke Atmaca ${ }^{1}$, Claudia Bavero ${ }^{1}$, Richard \\ McElreath $^{1}$, and Bret Beheim ${ }^{1}$ \\ ${ }^{1}$ Department of Human Behaviour, Ecology and Culture, Max Planck \\ Institute for Evolutionary Anthropology, Leipzig, Germany
}

November 4, 2020

\begin{abstract}
Reproducibility is integral to science, but difficult to achieve. We surveyed 560 empirical publications, published between 1955 and 2018 in the social learning literature, a research topic that spans animal behaviour, behavioural ecology, cultural evolution, and evolutionary psychology. Data was recoverable online or through direct data requests for $30 \%$ of this sample. Moreover, data recovery declines exponentially with time since publication, halving every 6 years, and up to every 9 years for human experimental data. When data for a publication can be recovered, we estimate a high probability of subsequent data usability (87\%), analytical clarity (97\%), and agreement of published results with reproduced findings $(96 \%)$. This corresponds to an overall rate of recovering data and reproducing results of $23 \%$, largely driven by unavailability or incompleteness of data. We thus outline clear measures to improve reproducibility of research on the ecology and evolution of social behaviour.
\end{abstract}




\section{${ }_{22}$ Introduction}

Scientific results often do not reproduce or replicate. This phenomenon is widely documented (Hardwicke et al., 2018; Vines et al., 2014; OSF, 2015), and calls for reform have spurred large-scale empirical and theoretical projects on the causes and consequences of non-replication and irreproducibility across scientific fields (Muthukrishna and Henrich, 2019; Klein et al., 2018; Smaldino and McElreath, 2016; Nissen et al., 2016; Higginson and Munafa, 2016; Maxwell et al., 2015). Community-wide demands such as mandatory data sharing (Hardwicke et al., 2018), preregistration of hypotheses (Nosek et al., 2018), and development of infrastructure (Munafò et al., 2017; Wilkinson et al., 2016; Ioannidis et al., 2014; McNutt, 2014) are now commonplace.

Of the goals of the growing open science movement, including transparency, integrity, and reliability, "replicability" has received considerable attention among the behavioural sciences, in part owing to the "replication crisis" of psychology (Pashler and Wagenmakers, 2012). By re-establishing results with new data (Goodman et al., 2016), replicability is critical to confirm the outcome of a proposed treatment or intervention, for example during clinical trials. Yet replication does not solely determine the quality of research and not all results may be expected to replicate (Devezer et al., 2019; Plesser, 2018).

For research in ecology and evolution, particularly for multigenerational studies of long-lived organisms such as humans, there are huge costs to data collection. Furthermore, it is impossible to engineer a repeated occurrence of a temporally specific phenomenon. An array of factors, from demographic transition, environmental variation, historical contingency, institutional dynamics such as market integration, and the specificities of field observation make it theoretically irrelevant and methodologically unfeasible to replicate the patterns that are often a focus of this research (Schnitzer and Carson, 2016).

While all research need not be replicable, it should be reproducible. Reproducibility means re-establishing findings using the same data and methods as in the original research (Goodman et al., 2016; Peng, 2011). This entails publicly available data, or data on request, along with unambiguous analysis procedures that, when repeated, reproduce results consistent with those originally reported. By ensuring the credibility and cogency of published work, streamlining workflows, increasing productivity and widening research recognition (Piwowar et al., 2007), reproducibility drives cumulative research. Yet reproducibility declines substantially with time since publication (Vines et al., 2014), and may fail even when enforced (Stodden et al., 2018; Baker et al., 2014). Despite recognition as a basic responsibility (Baker, 2016; Huang et al., 2012), reproducibility appears difficult to achieve.

Previous audits of reproducibility in the biological and behavioural sciences have focused on a single type of analysis (Gilbert et al., 2012), assessed the implementation of a journal's policy (Hardwicke et al., 2018), or evaluated specific aspects of reproducibility, such as availability of data (Vines et al., 2014) or code (Culina et al., 2020). Here, we employ a unified, general definition of reproducibility to characterise the reproducibility of a research literature. We sampled the literature on the topic of 


\section{${ }^{71}$ Results}

\section{Sample}

social learning - a multi-disciplinary research area that spans behavioural ecology, animal behaviour, cultural evolution and evolutionary psychology. Being methodologically and topically diverse, our sample permits a unique evaluation of the influence of different features of publications, such as constraints on sharing different types of data, and variation in research norms between sub-fields, on reproducibility.

We sampled empirical, quantitative research relevant to the topic social learning, including both experimental and observational work, with human and non-human study subjects. Our final sample included 560 empirical, quantitative papers published between 1955 and 2018. This comprised 446 experimental and 114 observational studies; 194 studies that included human subjects and 366 studies of non-human animals (nonhuman primates, birds, reptiles, fish, and small-bodied mammals). We identified 957 unique authors, each appearing on the author list of up to 49 papers in our sample, with a median number of one paper per author.

\section{Data recovery and reproducibility of results}

We recovered data for 167 studies, 30\% of our full sample of 560 papers. Data was available online - in supplementary information, data repositories or in the main article - for 62 of these papers (11\%). For an additional 105 papers, we were provided materials by authors of the publications. We sent emails requesting data and code to the authors of 473 publications, receiving a response in 315 cases $(56 \%$ of the total) (Figure 1). For 39 studies, authors indicated willingness to participate in our study, but we did not receive materials within our protocol time frame. In just 9 cases, authors indicated access to relevant files, but unwillingness to participate in our study. We obtained no information on the status of materials for 162 studies, online or through requests.

Of 167 studies that we were able to recover data for, we randomly selected 40 to estimate subsequent stages of reproducibility. Within these 40 studies, we identified a total of 111 results, with a median of 3 and a maximum of 6 results per study. Each result corresponded to an individually "citable" result from the publication abstract (see "Methods"). Using materials provided and details in the main article, we attempted to recreate at least one line of quantitative evidence that supported the result, such as a figure, table or model estimate.

Of 111 results, we determined the data to be usable and clear in 83 cases. This meant that we could access and open relevant data files, and confirm that the data corresponded to the stated result. Cases that failed included data files in incomprehensible formats, such as Microsoft Excel tables with multiply labelled or duplicated sheets. In other cases, data were missing, upon examination of materials. For example, the data for just one of two experiments of a publication were available to us, 
or the data included was aggregated for presentation and unusable for the purposes of reconstructing a result, or the relevant variable for a particular result was missing from, or unclear in the datasheets.

Of 83 results which corresponded to usable data, we determined the analysis to be clear and repeatable in 77 cases. An unclear analysis was for example a statistical technique (often non-parametric) that we could not find through a search of standard reference literature, or otherwise described a quantitative method that was not consistent with the kind of result being reported. This tended to be especially true of papers that employed "point-and-click" programs like Excel and SPSS, which involve a number of unrecorded, consecutive calculations. We did find that understanding specific analysis decisions based on descriptions in papers demanded a substantial amount of effort, and often we were able to devise a solution only by making inferential leaps.

When repeating the analyses for 77 results with clear analyses and data, we found the reconstructed result to correspond to the published findings in 73 cases. Here we focused on qualitative agreement of the stated and reconstructed finding, where appropriate. For example, for empirical frequencies or proportions, we confirmed results when they were quantitatively equivalent. For modelled results, however, we considered the level of detail in the original article; if the article reported only the significance of an effect, we looked for the same evidence. If the article reported the direction and magnitude of an effect, we confirmed that the direction and magnitude of our reconstructed result were consistent with this, if not numerically equivalent, to allow for sampling variation in estimation algorithms.

\section{A multi-stage model of reproducibility}

We characterised the staged, conditional process by which results of a paper can be successfully reproduced. Reproducibility depends sequentially on (1) data recoverability, the availability of data and other materials to attempt reproduction of analyses; (2) data usability, the completeness and clarity of data, when available; (3) analytical clarity, the adequacy of published reports for repeating analyses; and (4) agreement of reproduced results with published results, constituting a successful reproduction. Each stage is conditional upon previous stages. That is, if data can not be recovered, data usability and analytical clarity can not be assessed, and in turn if the data is unusable and the analysis unclear, the reproduction can not be attempted. When a result passes the first three stages, it may still fail to reproduce because it was originally misreported.

We thus estimated probabilities of reproducibility at each stage, conditional on previous stages, combining these to estimate an overall success rate for a publication (Table 1). That is,

$$
p(r)=\left(p_{1}\right)\left(p_{2}\right)\left(p_{3}\right)\left(p_{4}\right)
$$

where $r$ is the event that any particular result reproduces. We estimated $p_{1}$, the probability that data from a paper in our sample is recovered, to be 0.29 (CI: 0.27 
144 - 0.32). Accounting for unequal clustering of results within papers, we estimated

${ }_{145} p_{2}$, the conditional probability that data from a paper is usable, given that it is 146 recovered, to be 0.87 (CI: $0.62-0.98)$. We estimated $p_{3}$, the conditional probability 147 that analyses are understandable, given that data is available and usable, to be 0.97 ${ }_{148}(0.91-1.00)$. We estimated $p_{4}$, the conditional probability that reproduced results

agree with published results, given that data is available, usable, and analyses are understandable, to be $0.96(0.89-1.00)$. Thus, we estimate an overall success rate for a publication of $0.23(0.15-0.28)$. These results marginalise over the publication dates and distribution of study types within our sample.

\section{Data decay}

The availability of social learning data decays exponentially with time since publication (Figure 2, Panel A), indicated by the expected probability of finding material for any publication halving every 5.7 years on average (CI: $4.2-7.1$ ). Consequently, the probability of recovering data for studies published more than 20 years ago is close to zero (Figure 2, Panel A, expected probability less than 0.01 at $t=23$ years).

Decomposing data recovery by the type of data included, we found that the type of data in a study affects probability of recovery. Human experimental data decays most slowly, compared with other data types (Figure 2, Panel C). The estimated half-life for human experimental data, of 9.6 years $(6.1-12.8)$, is substantially larger than half-lives ranging from 5 to 6 years for other data types (human observational: 6.1 (2.0 - 9.2), non-human observational: 4.5 (3.8 - 5.3), non-human experimental: $6.5(4.0-9.0))$.

While age steeply influences data recovery, we found little to no effect of the influence of age on data usability, analytic clarity and reproduction of results. Accordingly, when decomposing the overall pubication success rate by age of publication, we estimate the probability in the year 2018 to be substantially higher than when marginalised across dates in the sample, at $0.68(0.50-0.86)$, rather than 0.23 , due to the higher probability of data recovery for recent publications.

\begin{tabular}{lrrrr} 
& mean & sd & $5.5 \%$ & $94.5 \%$ \\
\hline$p_{1}$ & 0.29 & 0.02 & 0.27 & 0.32 \\
$p_{2}$ & 0.87 & 0.13 & 0.62 & 0.98 \\
$p_{3}$ & 0.97 & 0.06 & 0.91 & 1.00 \\
$p_{4}$ & 0.96 & 0.05 & 0.89 & 1.00 \\
$p(r)$ & 0.23 & 0.04 & 0.15 & 0.28 \\
\hline
\end{tabular}

Table 1: Parameter, mean, standard deviation, and $94.5 \%$ compatibility interval: $p_{1}$ is the probability that data from a paper is recovered; $p_{2}$ is the conditional probability that data from a paper is usable, given that it is recovered; $p_{3}$ is the conditional probability that analyses are understandable, given that data is available and usable; $p_{4}$ is the conditional probability that results agree, given that data is available, usable, and analyses are understandable; and $p(r)$ is the overall probability of the event that results of a paper reproduce. 


\begin{tabular}{lrrrrrr} 
& mean & sd & $2.5 \%$ & $97.5 \%$ & $\mathrm{n} \_$eff & Rhat \\
\hline$\alpha_{1}$ & 0.81 & 0.07 & 0.66 & 0.95 & 12762.63 & 1.00 \\
$\alpha_{2}$ & 0.71 & 0.11 & 0.49 & 0.92 & 16382.66 & 1.00 \\
$\alpha_{3}$ & 0.86 & 0.07 & 0.70 & 0.97 & 16539.41 & 1.00 \\
$\alpha_{4}$ & 0.69 & 0.12 & 0.46 & 0.90 & 15315.34 & 1.00 \\
$\lambda_{1}$ & 0.07 & 0.02 & 0.04 & 0.10 & 13819.77 & 1.00 \\
$\lambda_{2}$ & 0.12 & 0.05 & 0.04 & 0.24 & 16612.93 & 1.00 \\
$\lambda_{3}$ & 0.15 & 0.02 & 0.12 & 0.18 & 18385.26 & 1.00 \\
$\lambda_{4}$ & 0.10 & 0.03 & 0.05 & 0.16 & 17368.21 & 1.00 \\
$\phi_{2}$ & 2.26 & 1.12 & -0.11 & 4.48 & 6882.41 & 1.00 \\
$\phi_{3}$ & 4.30 & 1.36 & 1.68 & 7.09 & 9696.87 & 1.00 \\
$\phi_{4}$ & 3.62 & 1.08 & 1.78 & 6.03 & 8991.47 & 1.00 \\
$\psi_{2}$ & 3.62 & 1.07 & 1.92 & 6.08 & 8391.14 & 1.00 \\
$\psi_{3}$ & 2.99 & 1.16 & 1.02 & 5.62 & 8138.95 & 1.00 \\
$\psi_{4}$ & 1.58 & 1.07 & 0.06 & 4.01 & 5577.50 & 1.00 \\
$\rho_{2}$ & 0.95 & 0.06 & 0.77 & 1.00 & 20314.40 & 1.00 \\
$\rho_{3}$ & 0.95 & 0.07 & 0.76 & 1.00 & 23544.27 & 1.00 \\
$\rho_{4}$ & 0.95 & 0.06 & 0.77 & 1.00 & 28133.96 & 1.00 \\
$v_{2}$ & 0.10 & 0.10 & 0.00 & 0.38 & 14811.30 & 1.00 \\
$v_{3}$ & 0.10 & 0.11 & 0.00 & 0.38 & 19790.13 & 1.00 \\
$v_{4}$ & 0.10 & 0.10 & 0.00 & 0.36 & 23691.31 & 1.00 \\
$\delta_{2}$ & 1.05 & 0.96 & 0.03 & 3.57 & 4692.80 & 1.00 \\
$\delta_{3}$ & 1.03 & 1.02 & 0.03 & 3.72 & 9457.77 & 1.00 \\
$\delta_{4}$ & 0.67 & 0.67 & 0.02 & 2.45 & 15004.94 & 1.00 \\
\hline & & & & & &
\end{tabular}

Table 2: Parameter, mean, standard deviation, 94.5\% compatibility interval, number of effective samples and the $\widehat{R}$ convergence diagnostic values for main model parameters.

\section{${ }_{172}$ Discussion}

Under one in four reproduction attempts in the social learning literature succeed. Our sample and analysis indicate that reasonable effort to recover data will fail for about 70 of 100 potential papers. Conditional on recovering data, we expect a further 6 to 7 papers to fail to reproduce, because of unclear data, ambiguous analysis methods or reanalysis disagreements. This corresponds to a successful reproduction for just under 25 of a potentially sampled 100 papers.

Data unavailability is by far the largest barrier to reproducibility, indicated by a steep decline in data recovery of about $11 \%$ per year, compounded with just $87 \%$ of data being usable upon recovery. Sharing data in the behavioural sciences was neither normative nor feasible until recently. Before the proliferation of open-source software tools (Wilkinson et al., 2016), data was often stored in outdated or proprietary formats. Frequently, replies to our requests included the phrase, "unfortunately, that study was X computers ago."

To extend data lifetime, we require institutional infrastructures, because individ- 
ual researchers retire, and data accumulates over a career. Journals have adopted data sharing policies (Simmons, 2017; Stodden et al., 2013; Hanson et al., 2011), but these face major challenges - proper enforcement (Roche, 2017; Baker et al., 2014; McCullough et al., 2008), including appraising files before publication (Hardwicke et al., 2018), empowering reviewers (Parker et al., 2018), and expanded hosting for analysis code as well as data files (Mislan et al., 2016). It should become common for research institutions or cross-institutional collaborations to invest in hiring data managers and developing bespoke data processing pipelines.

Most researchers publishing in ecology and evolution are fluent in computational tools, but not necessarily in data science skills. The use of plaintext data formats ensures stable, long-term accessibility of data, and free hosting services are available through the Open Science Framework, GitHub, and Data Dryad. Numerous resources are available, being developed, and increasingly utilised to improve data science fluency, such as Software Carpentry (http://software-carpentry.org), R for Data Science (Wickham and Grolemund, 2017), The Practice of Reproducible Science (Kitzes et al., 2017), and FAIR (Findable, Accessible, Interoperable, and Reusable (Wilkinson et al., 2016)), among others (Culina et al., 2018; Ihle et al., 2017; Whitlock, 2011).

Moreover, maintaining data provenance is central to reproducibility. If data are to be re-used, the entire history of the data and its origins, from data collection, transcription, coding, transformation and analysis should be documented and archived. This is facilitated by version control systems (e.g. git). Data that is too raw, or too processed, is difficult to re-use. Re-processing raw video or audio files, while theoretically possible, can be prohibitively difficult, demanding an unreasonable amount of effort. Yet, highly processed data can fail to correspond to analyses presented in an article, for example when aggregated into categories, rather than a single entry per observation. The meaning of variables can become impossible to decipher over time, given frequent updates to variable descriptions for presentation purposes, or creation of new, very similar variables. This is especially true for longitudinal data, where new data is continuously added, thus easily conflated with older data. That data be archived, in a tabular format, along with a data dictionary or variable key in the language of publication (for our study, English) is not trivial practice.

Authors of papers utilising longitudinal, observational datasets expressed fears that published data may be wrongly re-interpreted and thus bolster false or contradictory analyses. Data is extremely costly to generate. Still, a fundamental feature of scientific work is the ability to independently draw conclusions about proposed findings. Numerous solutions to issues of data privacy and rightful credit exist, including data publishing embargoes, password-protected databases, and citable DOIs. A related concern is that data is extremely costly to prepare for sharing upon request. Yet ostensibly, materials should be ready to share at the review stage of the publication process, as reviewers regularly request access to the data. If data are well-archived, well-documented and made available at the time of publication, it should demand negligible time and effort to deal with, or obviate the need for, a data request.

While data lifetime and interpretability are undoubtedly the primary barrier to reproducibility, clarity of analyses themselves to hinder reproducibility. Recent anal- 
yses are often documented in statistical scripting languages such as R, Stata, Python, Mathematica and Matlab, but authors are hesitant to make code public. Even when non-functional, code (in any programming language) greatly facilitated our ability to reconstruct results. Messy code is better than no code (Barnes, 2010), as it can document data manipulation or exclusion that is otherwise opaque, clarify the sequence of analyses conducted, and record any algorithm assumptions. These details are likely too complex to compress, even into a detailed supplement. Moreover, an early commitment to share code can improve the way that code is written, encouraging the use of "linting" for code readability (Wickham, 2015), and explanatory code comments, facilitating reproducibility.

A relatively high rate of response to a survey, even when materials were not recoverable, and general support for the goals of our study, is consistent with previous work that indicates researchers readily accept reproducible norms and reward reproducible practices (Wallis et al., 2013; Kidwell et al., 2016; Campbell et al., 2019). Our finding that human experimental data has a longer lifetime than other data types could be indicative of earlier adoption of research norms in the field of psychology, which may be more attuned to open science than other research areas; the "Replication Crisis" has also been called "the Credibility Revolution" (Vazire, 2018). The growing recognition of the importance of reproducibility is encouraging for researchers, particularly junior scholars, who often consider making requests for materials from published work.

The decay rate of social learning data, while alarming, is not unique. Our estimate of approximately $30 \%$ data recoverability resembles findings of $38 \%$ (Vanpaemel et al., 2015), 32\% (Hardwicke and Ioannidis, 2018), 26\% (Wicherts et al., 2006), and 19\% (Vines et al., 2014). Our estimate of results reproducibility, conditional on previous stages, is difficult to quantitatively compare against similar audits, which have found reproducibility of results to be anywhere between 83\% (Andrew et al., 2015), 70\% (Gilbert et al., 2012), and 1.1\% (Stagge et al., 2019) of surveyed publications. Because the skillsets of a reasonable researcher can only be defined relative to the norms of a particular scholarly community, any reproducibility effort has to be defined in similar relative terms. Indeed, our protocol focused on qualitative agreement with results stated in the publication abstract, focusing on findings that will likely be cited by future work.

Reproducibility of empirical work is a minimum requirement for research in our field - a necessary precursor to replication, meta-analysis or further theory development. Our research implies that the rapid adoption of reproducible norms and tools of data sharing will slow data decay tremendously, such that a study performed in a decade will show a very different decay rate, and correspondingly a much higher reproducibility rate, than estimated here. 


\section{Methods}

\section{Sample}

We downloaded citations over a period of 5 months (February to July 2018), identifying published articles and book chapters through Google Scholar searches for phrases such as "social learning", "animal culture", "cumulative culture", "cultural transmission", "payoff bias", "diffusion", "imitation", "innovation", "social information use", "teaching", "tool-use", and "conformity", and through forward and backward citation to identify additional studies.

For each publication, we assigned a unique identification key and recorded the DOI, the title, year of publication, and the journal the article was published in. We coded features of the study, including the study species and the type of study design (experimental or observational), and whether supplementary material was published along with the article. We also searched each manuscript for a statement about the location of materials that would help reproduce results, using a list of keywords ("code", "available", "provided", "data", "analysis", "material", "reproduce", "results"). If the statement about materials pointed to an external data repository or website, we recorded this, and downloaded any available materials.

We wrote to corresponding authors, or first authors when corresponding authors were not identifiable, to request data and analytical code. For research published more than four years ago, we performed a brief Google search to identify a morerecent email address of authors. We sent requests in batches over five months (June to October 2018), with a follow-up reminder approximately five weeks after a first request.

We also contacted authors if the available material was clearly incomplete, or if we would benefit from accessing additional material (e.g. code or data in a different format, such as when data were included as a table within the article). We did not write data requests if we recorded that materials were complete (including code as well as data) on a first look $(\mathrm{n}=11)$, we were aware the corresponding author was no longer alive $(\mathrm{n}=4)$, we could not locate an email address $(\mathrm{n}=20)$, and, in a single case, because we knew from previous correspondence that the materials would be inaccessible.

Post-correspondence, we surveyed the materials downloaded and the email correspondence to code the status of materials as one of "material available online", "material received", "material lost", "material inaccessible to author or requester within timeframe", "no information on material (no response)", "no information on material (no request)".

\section{Protocol for evaluating results}

We evaluated data usability, analytic clarity and results reproducibility for a random subset of studies for which we recovered materials, because of constraints on the number of complete reproductions we could feasibly complete. We selected 40 studies randomly (see https://github.com/rianaminocher/reproducibility-analysis for 
details on a rough computation of power).

For each paper in the subset, we identified individually citable results from the abstract of the publication. We located corresponding in-text references for each result, to establish evidence for each in the form of figures, tables or estimates. For a single paper that did not contain an abstract, we identified the main results from the results section of the paper. If the data provided were not usable or complete, (e.g. too cryptic, raw, or insufficiently annotated), the point of failure for that result was at stage 2, 'data unclear'. If the data were usable, but the analysis was unclear, for example being under-described in the text, or too complicated or novel to implement without analytical code or substantial support, the result failed at stage 3, 'analysis unclear'.

If a result passed the first, second, and third stages of the model, we considered whether we could successfully reproduce the result. If our reconstructed result differed from the result in the paper, such as reversing the direction of the stated effect, we coded the cause of failure to be 'reanalysis result differs'. A reproduction was successful if we recreated at least one line of evidence per result, allowing for non-exact numerical reproductions caused by differences in software or estimation algorithms. We reproduced all analyses in $\mathrm{R}$, even if originally conducted in alternative software, such as SPSS. An example of our reproduction protocol is available at https:// github.com/rianaminocher/reproducibility-example.

As we verified the materials we downloaded, and continued to correspond with authors, we consequently re-categorised some studies that had initially been coded as having "material available" to "material lost" or "material not readily available". We did this in 11 cases. In 9 of 11 of these cases, we had classified the publication as including material, because descriptive data appeared available in the article pdf. For example, an empirical paper describing patterns of behaviour across individuals may have included tables detailing frequency of behaviours within groups, within the text.

We found that the original data for some of these "descriptive" publications, which may have included field observations, does not necessarily exist and may not be accessible to us. We treated these results as we treated "descriptive" results across the sample. We assessed reproducibility of descriptive results by identifying whether individual-level or observation-level data to support the result was available in text. If we were able to dis-aggregate tabulated frequencies of behaviour to produce the reported result, we coded the result as reproducible.

\section{Statistical analysis}

Let $x_{s, i}$ be the outcome of stage $s \in\{1,2,3,4\}$ for publication $i$. In the first stage, $s=1$, each publication $i$ has $x_{1, i}=1$ if data were recovered and $x_{1, i}=0$ otherwise. We assume the probability of data recovery decays exponentially with time since publication. In statistical notation: 


$$
\begin{aligned}
x_{1, i} & \sim \operatorname{Bernoulli}\left(p_{1, i}\right) \\
p_{1, i} & =\alpha_{Y[i]} \exp \left(-\lambda_{Y[i]} v_{i}\right)
\end{aligned}
$$

where $v_{i}$ is the number of years since publication, $\alpha$ is the probability of recovery at time of publication, and $\lambda$ is the rate of decay. We allow both $\alpha$ and $\lambda$ to vary with other features of the paper, labeled here as the study type $Y_{i}$. Study type is a combination of species studied and study design.

Data recovery may be affected by any large number of errors, including loss of files, retirement of archives, and technological change, which results in an exponential decay of probability with time since publication. Data including human and non-human subjects are likely constrained by different ethical regulations. Concomitantly, experimental data may be easier than observational data to share, having been generated in a specific format and unlikely to be re-used or re-analysed by the experimenter, in contrast to observational data, which is often used repeatedly, to investigate different research questions.

When $x_{1, i} \neq 0$, the second stage, data usability, is assessed; $x_{2, i} \neq 0$, the third stage, analytical reproducibility, is assessed; and $x_{3, i} \neq 0$, the final stage, successful reproduction, is assessed. The statistical models for these stages are identical, but with independent parameters. For $s=2, s=3$ and $s=4$, the rate of reproducibility is a function of the paper's publication date $v_{i}$ as in the first stage. We cluster on publication and model the variation among results in each publication. In statistical notation, for $s=2, s=3$ or $s=4$ :

$$
\begin{aligned}
x_{s, i} & \sim \operatorname{Binomial}\left(n_{i}, p_{s, i}\right) \\
\operatorname{logit}\left(p_{s, i}\right) & =\phi_{s}+\beta_{s, i}+\gamma_{s, v[i]}
\end{aligned}
$$

We do not assume any particular functional form for the relationship with $v_{i}$, instead using a Gaussian process smoother.

$$
\begin{aligned}
\gamma_{s, j} & \sim \text { Multi.Normal.Cholesky }\left(0, \delta_{s} \mathbf{L}_{s}\right) \\
\kappa_{s, m n} & \left.=\rho_{s} \exp \left(-v_{s} D_{m n}^{2}\right)\right)
\end{aligned}
$$

Here, $\mathbf{L}_{s}$ is a factor given by the Cholesky decomposition of the correlation matrix $\kappa$ and $\delta_{s}$ scales variance. The correlation matrix $\kappa$ is defined by the distance decay function in which $D_{m n}^{2}$ is the squared, normalized distance between publication dates $m$ and $n, \rho_{s}$ is the maximum correlation between neighbouring age parameters, and $v_{s}$ is the rate at which the correlation decays with increasing distance between publication dates.

We used the following priors to constrain parameters to plausible values. 


$$
\begin{aligned}
\alpha_{k} & \sim \operatorname{Beta}(4,2) \\
\lambda_{k} & \sim \log \operatorname{Normal}(-2,1) \\
\phi_{s} & \sim \operatorname{Normal}(0,2.5) \\
\beta_{s, i} & \sim \operatorname{Normal}\left(0, \psi_{s}\right) \\
\psi_{s} & \sim \operatorname{Exponential}(1) \\
\rho_{s} & \sim \operatorname{Beta}(10,1 / 2) \\
v_{s} & \sim \operatorname{Exponential}(10) \\
\delta_{s} & \sim \operatorname{Exponential}(1)
\end{aligned}
$$

These priors represent an extremely wide range of decay dynamics. We validate the sensibility of these priors using prior predictive simulations (see https: //github.com/rianaminocher/reproducibility-analysis for details). We conducted analyses in R (4.0.3, R Core Team (2020)). We fit our model using the Stan engine, implemented in R with rstan (2.21.2, Stan Development Team (2019)). Results presented are summaries of 10,000 iterations of 4 chains. We assessed convergence and mixing with the 4th version of the $\widehat{R}$ convergence diagnostic as implemented in Stan (Gelman et al., 2013), the estimate of the autocorrelationadjusted number of samples (n_eff), and visual inspection of the trace plots of all chains. We provide data and analysis code for reproducing our reported results at https://github.com/rianaminocher/reproducibility-analysis.

\section{Limitations of study design}

To some extent, our generative model of reproducibility is confounded, as we cannot infer possible states of the data or result beyond the point of failure. This means that all failure states are logically prior to the final one, of disagreement between published findings and reproduced results, and might therefore mask potential cases of failure at this stage. Further to this, to estimate the overall reproducibility of our sample, we did not consider sets of papers which failed at any stage. Our study design prohibits this. Consider the following counter-factual reality - we identify a result that failed to reproduce, because the data were lost. Then we go back in time and intervene, such that the data are saved. The expected probability that this result is reproducible may not be the same as a result for which the data were recovered, in our reality, because there may be associations between data recoverability and subsequent stages of reproducibility. We have only measured subsequent stages of reproducibility for results for which data exist, that is, conditionally, so we cannot estimate the causal influence of results reproducibility unconditionally.

A major challenge to establishing a useful estimate of reproducibility is that some researchers may succeed to reproduce a result where other researchers fail. This can be because of a lack of access to necessary software (proprietary programs) or hardware (server clusters), or a lack of necessary skill or knowledge for a specialised 
analysis. Given this, there is no universal standard with which to label a result "non-reproducible".

One way to operationalise reproducibility is through expectations of access to software, hardware, skills and knowledge for any particular reproduction. Throughout our study, we attempted to remain conscious of the time, effort and skillset that a typical early-career researcher in the evolutionary behavioural sciences might invest in a reanalysis ("the reasonable researcher criterion"). We sampled papers published in English, used institutional access to software and server clusters, researched methods we were unfamiliar with, and endeavoured to correspond with authors of publications in a formal, professional tone. This is central to our definition of a successful reproduction.

Our assessment of reproducibility does not appraise the overall quality of research. In reconstructing analyses exactly as presented in the paper, we did not critique appropriateness of methodological or analytical choices for particular results, even when we ourselves might have collected or processed the data differently. Such a reproduction procedure is complementary to, but no substitute for, criticism of the scientific value of a published result or approach.

\section{Acknowledgements}

We thank Anne Büchner, Leonie Ette and Kristina Kunze for collecting data, Cody T. Ross for consulting on analyses and model parameterisation, and the Department of Human Behaviour, Ecology and Culture at the Max Planck Institute for Evolutionary Anthropology for helpful discussion and feedback on the project at various stages.

\section{Competing interests}

We declare no competing interests.

\section{References}

Andrew, R. L., Albert, A. Y., Renaut, S., Rennison, D. J., Bock, D. G., and Vines, T. (2015). Assessing the reproducibility of discriminant function analyses. PeerJ, 3:e1137.

Baker, D., Lidster, K., Sottomayor, A., and Amor, S. (2014). Two years later: journals are not yet enforcing the arrive guidelines on reporting standards for pre-clinical animal studies. PLoS Biology, 12(1):e1001756.

Baker, M. (2016). Reproducibility crisis? Nature, 533(26):353-366.

Barnes, N. (2010). Publish your computer code: it is good enough. Nature News, 467(7317):753-753. 
Campbell, H. A., Micheli-Campbell, M. A., and Udyawer, V. (2019). Early career researchers embrace data sharing. Trends in Ecology \&f Evolution, 34(2):95-98.

Culina, A., Baglioni, M., Crowther, T. W., Visser, M. E., Woutersen-Windhouwer, S., and Manghi, P. (2018). Navigating the unfolding open data landscape in ecology and evolution. Nature Ecology \&6 Evolution, 2(3):420-426.

Culina, A., van den Berg, I., Evans, S., and Sánchez-Tójar, A. (2020). Low availability of code in ecology: A call for urgent action. PLoS Biology, 18(7):e3000763.

Devezer, B., Nardin, L. G., Baumgaertner, B., and Buzbas, E. O. (2019). Scientific discovery in a model-centric framework: Reproducibility, innovation, and epistemic diversity. PLoS One, 14(5).

Gelman, A., Carlin, J. B., Stern, H. S., Dunson, D. B., Vehtari, A., and Rubin, D. B. (2013). Bayesian Data Analysis. Chapman and Hall/CRC.

Gilbert, K. J., Andrew, R. L., Bock, D. G., Franklin, M. T., Kane, N. C., Moore, J.-S., Moyers, B. T., Renaut, S., Rennison, D. J., Veen, T., et al. (2012). Recommendations for utilizing and reporting population genetic analyses: the reproducibility of genetic clustering using the program structure. Molecular Ecology, 21(20):49254930 .

Goodman, S. N., Fanelli, D., and Ioannidis, J. P. A. (2016). What does research reproducibility mean? Science Translational Medicine, 8(341):341ps12.

Hanson, B., Sugden, A., and Alberts, B. (2011). Making data maximally available. Science, 331(6018):649.

Hardwicke, T. E. and Ioannidis, J. P. (2018). Populating the data ark: An attempt to retrieve, preserve, and liberate data from the most highly-cited psychology and psychiatry articles. PLoS One, 13(8).

Hardwicke, T. E., Mathur, M. B., MacDonald, K., Nilsonne, G., Banks, G. C., Kidwell, M. C., Hofelich Mohr, A., Clayton, E., Yoon, E. J., Henry Tessler, M., et al. (2018). Data availability, reusability, and analytic reproducibility: Evaluating the impact of a mandatory open data policy at the journal cognition. Royal Society Open Science, 5(8):180448.

Higginson, A. D. and Munafa, M. R. (2016). Current incentives for scientists lead to underpowered studies with erroneous conclusions. PLoS Biology, 14(11).

Huang, X., Hawkins, B. A., Lei, F., Miller, G. L., Favret, C., Zhang, R., and Qiao, G. (2012). Willing or unwilling to share primary biodiversity data: results and implications of an international survey. Conservation Letters, 5(5):399-406.

Ihle, M., Winney, I. S., Krystalli, A., and Croucher, M. (2017). Striving for transparent and credible research: Practical guidelines for behavioral ecologists. Behavioral Ecology, 28(2):348-354. 
Ioannidis, J. P., Greenland, S., Hlatky, M. A., Khoury, M. J., Macleod, M. R., Moher, D., Schulz, K. F., and Tibshirani, R. (2014). Increasing value and reducing waste in research design, conduct, and analysis. The Lancet, 383(9912):166-175.

Kidwell, M. C., Lazarević, L. B., Baranski, E., Hardwicke, T. E., Piechowski, S., Falkenberg, L.-S., Kennett, C., Slowik, A., Sonnleitner, C., Hess-Holden, C., et al. (2016). Badges to acknowledge open practices: A simple, low-cost, effective method for increasing transparency. PLoS Biology, 14(5).

Kitzes, J., Turek, D., and Deniz, F. (2017). The practice of reproducible research: case studies and lessons from the data-intensive sciences. University of California Press.

Klein, R. A., Vianello, M., Hasselman, F., Adams, B. G., Adams Jr, R. B., Alper, S., Aveyard, M., Axt, J. R., Babalola, M. T., Bahník, Š., et al. (2018). Many labs 2: Investigating variation in replicability across samples and settings. Advances in Methods and Practices in Psychological Science, 1(4):443-490.

Maxwell, S. E., Lau, M. Y., and Howard, G. S. (2015). Is psychology suffering from a replication crisis? what does "failure to replicate" really mean? American Psychologist, 70(6):487.

McCullough, B. D., McGeary, K. A., and Harrison, T. D. (2008). Do economics journal archives promote replicable research? Canadian Journal of Economics/ Revue canadienne d'économique, 41(4):1406-1420.

McNutt, M. (2014). Journals unite for reproducibility.

Mislan, K., Heer, J. M., and White, E. P. (2016). Elevating the status of code in ecology. Trends in Ecology 85 Evolution, 31(1):4-7.

Munafò, M. R., Nosek, B. A., Bishop, D. V., Button, K. S., Chambers, C. D., Du Sert, N. P., Simonsohn, U., Wagenmakers, E.-J., Ware, J. J., and Ioannidis, J. P. (2017). A manifesto for reproducible science. Nature Human Behaviour, 1(1):0021.

Muthukrishna, M. and Henrich, J. (2019). A problem in theory. Nature Human Behaviour, 3(3):221-229.

Nissen, S. B., Magidson, T., Gross, K., and Bergstrom, C. T. (2016). Publication bias and the canonization of false facts. eLife, 5:e21451. arXiv: 1609.00494.

Nosek, B. A., Ebersole, C. R., DeHaven, A. C., and Mellor, D. T. (2018). The preregistration revolution. Proceedings of the National Academy of Sciences, 115(11):26002606.

OSF (2015). Estimating the reproducibility of psychological science. Science, 349(6251). 
Parker, T. H., Griffith, S. C., Bronstein, J. L., Fidler, F., Foster, S., Fraser, H., Forstmeier, W., Gurevitch, J., Koricheva, J., Seppelt, R., et al. (2018). Empowering peer reviewers with a checklist to improve transparency. Nature Ecology 8 Evolution, 2(6):929.

Pashler, H. and Wagenmakers, E.-J. (2012). Editors' introduction to the special section on replicability in psychological science: A crisis of confidence? Perspectives on Psychological Science, 7(6):528-530.

Peng, R. D. (2011). Reproducible research in computational science. Science, $334(6060): 1226-1227$.

Piwowar, H. A., Day, R. S., and Fridsma, D. B. (2007). Sharing detailed research data is associated with increased citation rate. PLoS One, 2(3):e308.

Plesser, H. E. (2018). Reproducibility vs. replicability: a brief history of a confused terminology. Frontiers in Neuroinformatics, 11:76.

R Core Team (2020). R: A Language and Environment for Statistical Computing. R Foundation for Statistical Computing, Vienna, Austria.

Roche, D. G. (2017). Evaluating Science's open-data policy. Science, 357(6352):654.

Schnitzer, S. A. and Carson, W. P. (2016). Would ecology fail the repeatability test? BioScience, 66(2):98-99.

Simmons, L. W. (2017). Guidelines for Transparency and Openness (TOP). Behavioral Ecology, 28(2):347-347.

Smaldino, P. E. and McElreath, R. (2016). The natural selection of bad science. Royal Society Open Science, 3(9):160384. arXiv: 1605.09511.

Stagge, J. H., Rosenberg, D. E., Abdallah, A. M., Akbar, H., Attallah, N. A., and James, R. (2019). Assessing data availability and research reproducibility in hydrology and water resources. Scientific Data, 6:190030.

Stan Development Team (2019). RStan: the R interface to Stan. R package version 2.19.2.

Stodden, V., Guo, P., and Ma, Z. (2013). Toward reproducible computational research: an empirical analysis of data and code policy adoption by journals. PLoS One, 8(6):e67111.

Stodden, V., Seiler, J., and Ma, Z. (2018). An empirical analysis of journal policy effectiveness for computational reproducibility. Proceedings of the National Academy of Sciences, 115(11):2584-2589.

Vanpaemel, W., Vermorgen, M., Deriemaecker, L., and Storms, G. (2015). Are we wasting a good crisis? the availability of psychological research data after the storm. Collabra, 1(1):1-5. 
Vazire, S. (2018). Implications of the credibility revolution for productivity, creativity, and progress. Perspectives on Psychological Science, 13(4):411-417.

Vines, T. H., Albert, A. Y. K., Andrew, R. L., Débarre, F., Bock, D. G., Franklin, M. T., Gilbert, K. J., Moore, J. S., Renaut, S., and Rennison, D. J. (2014). The availability of research data declines rapidly with article age. Current Biology, 24(1):94-97.

Wallis, J. C., Rolando, E., and Borgman, C. L. (2013). If we share data, will anyone use them? data sharing and reuse in the long tail of science and technology. PLoS One, $8(7)$.

Whitlock, M. C. (2011). Data archiving in ecology and evolution: best practices. Trends in Ecology \&6 Evolution, 26(2):61-65.

Wicherts, J. M., Borsboom, D., Kats, J., and Molenaar, D. (2006). The poor availability of psychological research data for reanalysis. American Psychologist, 61(7):726.

Wickham, H. (2015). R packages: organize, test, document, and share your code. O'Reilly Media, Inc.

Wickham, H. and Grolemund, G. (2017). $R$ for Data Science: Import, Tidy, Transform, Visualize, and Model Data. O’Reilly Media, Inc., 1st edition.

Wilkinson, M. D., Dumontier, M., Aalbersberg, I. J., Appleton, G., Axton, M., Baak, A., Blomberg, N., Boiten, J.-W., da Silva Santos, L. B., Bourne, P. E., et al. (2016). The fair guiding principles for scientific data management and stewardship. Scientific Data, 3. 

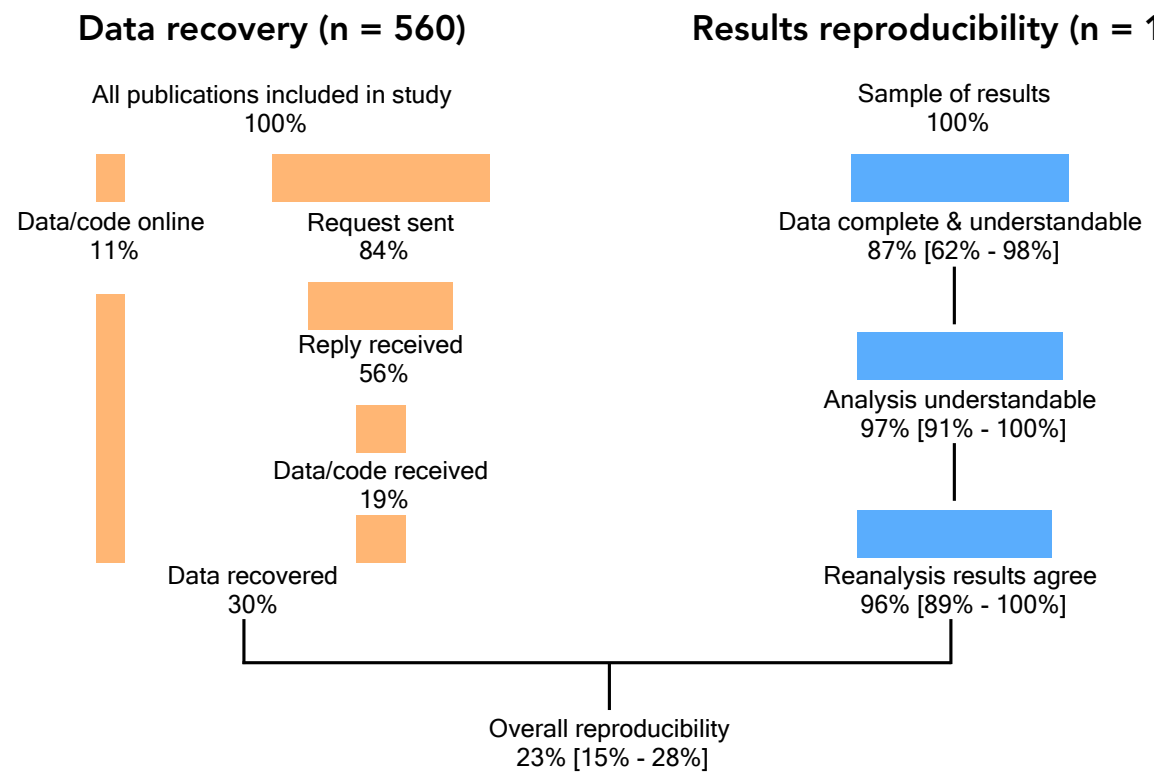

Figure 1: Data recovery: Of 560 publications surveyed, we recovered data online for $62(11 \%)$. We sent requests to authors of 473 of the remaining papers (84\% of the sample), and received a reply in 315 cases (56\%). Through correspondence, we received materials for an additional 105 publications (19\%). We thus categorised a total of 167 studies with materials available, $30 \%$ of our initial sample. Results reproducibility: To evaluate subsequent stages of reproducibility, we randomly sampled 40 of these 167 publications. From 111 results we identified, we estimated the probability of data usability, given materials are available, to be $87 \%$ (83/111 results). The probability of analytical clarity, given data is available and usable, is estimated to be $97 \%$ (77/83 results). The probability that reproduced results correspond to reported results, given data is available and usable, and analysis clear, is estimated to be $96 \%$ (73/77 results). This results in an overall probability of reproducibility across all stages of $23 \%$. 

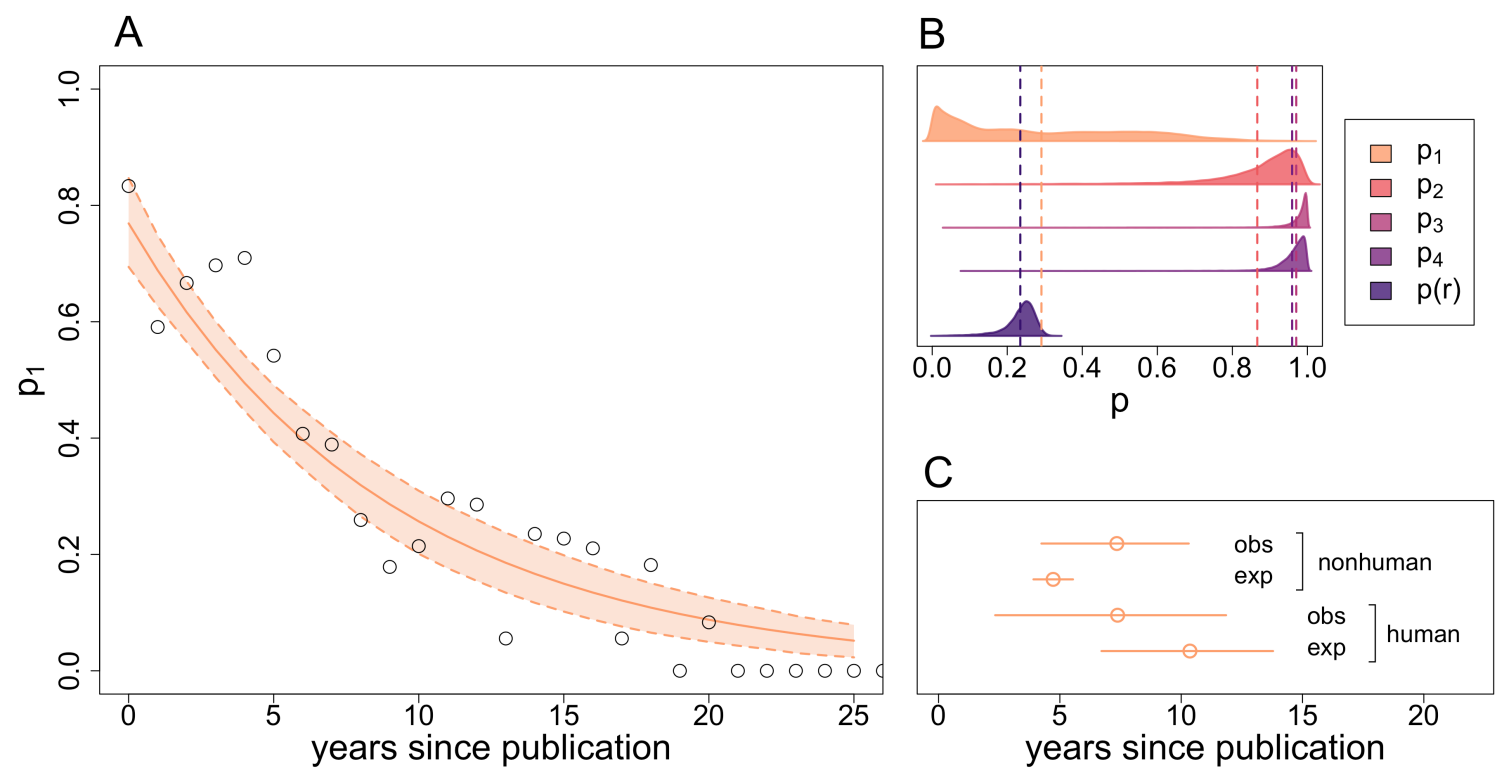

Figure 2: A: The predicted probability of data recovery declines exponentially with increasing time since publication, halving every 6 years. The solid line plots the expected exponential decay curve, shaded interval between dotted lines shows the $89 \%$ compatibility interval and empty circles plot the observed data, i.e. raw proportion of studies, for each year, for which we obtained materials. B: Posterior prediction densities, marginalised across ages of papers in the sample, for $p_{1}$, the probability of data recovery; $p_{2}$, the probability of data usability, conditional on recovery; $p_{3}$, the probability analytical clarity, conditional on data usability and recovery; $p_{4}$ the probability of reproduction, conditional on analytical clarity, data recovery and usability; and $p(r)$, the combined probability of reproducibility across all four stages of data recovery, usability, analytical clarity and final reproduction. C: The data half-life, decomposed by study type, indicates that human experimental data decays most slowly. 


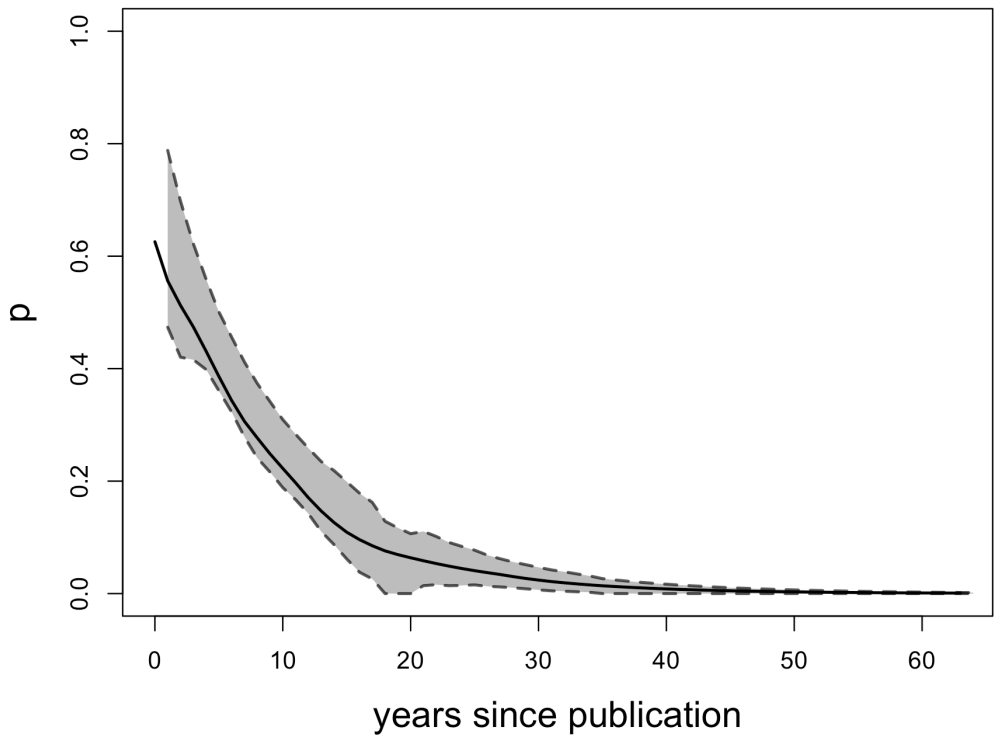

Figure 3: Figure 2A, Supplement: The overall probability of reproduction decays with publication age, owing to the exponential decline in data recovery. The solid line plots the expected probabilities by publication age, and the dotted lines and shaded interval plot the $89 \%$ interval. 